\title{
Laser-induced incandescence in aqueous carbon black suspensions: the role of particle vaporization
}

\author{
Ju.Ju. Rulik, N.M. Mikhailenko, S.E. Zelensky, A.S. Kolesnik \\ Taras Shevchenko Kyiv National University, Physics Department \\ 6, Academician Glushkov prospect, 03680 Kyiv, Ukraine, e-mail: zele@univ.kiev.ua
}

\begin{abstract}
Non-linear properties and the kinetics of laser-induced incandescence in aqueous carbon black suspensions are investigated. For explanation of the observed properties, a model, which takes into account a decrease of the size of particles caused by their vaporization under laser irradiation, is proposed.
\end{abstract}

Keywords: laser-induced incandescence, carbon black suspension, particle vaporization.

Manuscript received 01.02.07; accepted for publication 24.04.07; published online 19.10.07.

\section{Introduction}

Laser-induced incandescence (LII) is a distinctive feature of the interaction of powerful laser radiation with fine-grained matter. LII appears at the quick overheating of microparticles irradiated by nanosecond laser pulses. At a moderate level of laser power density, below $50 \mathrm{MW} \cdot \mathrm{cm}^{2}$, the local temperature of submicron-sized particles is estimated to reach thousands of Kelvins. LII is observed with submicron particles suspended in gases and liquids or included into solid-state matrices. LII in the gaseous phase is investigated in detail and is employed as a method to control the content of soot in exhaust flows and in flames of different types [1-7]. The mechanism of LII in gases presumes the quick heating of a soot particle up to the temperature of carbon vaporization (about $4000 \mathrm{~K}$ ). Upon reaching this value, the further increase of the particle temperature slows down and the energy received from laser radiation is spent almost completely on the particle vaporization. As a result, the excitation curve of LII has a plateau $[2,6]$. In particular, this plateau enables the use of LII for soot diagnostics. The spectra of LII are broad and structureless, ranging from ultraviolet to infrared, and they can be fitted by Planck's black-body emission formula. In the literature, there are the reports on laser-induced transformations of the internal structure of irradiated particles [4].

As regards particles suspended in condensed media, LII was also observed with uncontrollable inclusions in borate glasses [8] and with black pigment particles in water (gouache, ink) [9-11]. LII in condensed matter is observed at moderate intensities of laser excitation in the range of $5-50 \mathrm{MW} \cdot \mathrm{cm}^{-2}$. For excitation of LII, the laser wavelength is of secondary importance, as well as the particle chemical composition. If the infrared beam of an YAG: $\mathrm{Nd}^{3+}$ laser is chosen as a source of excitation, then LII is clearly seen to the naked eye as a white uniform track. In the case of infrared laser excitation, a substantial portion of the LII spectrum is contained in the shortwavelength region relative to the wavelength of excitation, i.e. this portion of LII can be considered as a typical instance of anti-Stokes emission. It is worth noting that this emission possesses non-linear properties.

LII is an additional source of errors in laser spectroscopy. For measuring weak optical signals against a background of laser scattering, an investigator should be careful in the interpretation of the signals detected. As is noted in [12], LII in porous silicon was mistakenly interpreted in some cases as a luminescence.

The thorough theoretical description of LII on the microscopic level appears to be a complicated problem which is now far from being solved. For laser-heated particles in gases, it was determined that the main mechanism of energy loss is the vaporization of a particle material [17]. Obviously, one of the basic distinctions between LII in condensed matter and LII in gases is the mechanism of particle energy dissipation. The properties of LII in glasses and aqueous suspensions at moderate intensities of laser excitation are analyzed in $[8,10]$. There, assuming that thermal conduction into the surrounding medium is the dominant mechanism of particle energy dissipation, a satisfactory explanation is obtained for most of the experimentally observed LII data. In addition, with the same assumption, a method is proposed for the particle temperature estimation using the results of measuring the LII emission intensity as a function of laser excitation intensity. 
As is known [9, 13-20], LII in suspensions of lightabsorbing particles and carbon nanotubes is accompanied by a significant decrease of optical transmittance with increase in the laser power (the effect of optical limiting). Two main mechanisms of optical limiting in suspensions are considered [9]. First, optical limiting in suspensions is caused by intensive vaporization of a liquid (water, ethanol) around a laser-heated particle. Within a few nanoseconds of the laser action on particles, a gaseous shell (a bubble) grows around (or in the vicinity of) the particle. The size of the gaseous shell is comparable to the size of the particle (approximately $\left.10^{-7} \ldots 10^{-6} \mathrm{~m}\right)$ or a few times larger. Numerical simulations [20] indicate that the vaporization of the surrounding liquid is not a dominant mechanism of particle energy loss: only a small fraction of the particle energy $(0.5 \ldots 1 \%)$ is spent on the vaporization. Nevertheless, the formation of microbubbles causes a significant increase of the light scattering in the suspension, and therefore the optical transmittance of the laser-irradiated suspension decreases considerably. The second mechanism of optical limiting in suspensions is the microplasma formation around heated particles in the laser field $[9,13]$. Unfortunately, relative contributions of the mentioned mechanisms to the particle energy general balance and to the effect of optical limiting are not known.

The present paper is devoted to the experimental investigation of LII in the aqueous suspensions of black submicron particles. The characteristics of LII are investigated, which enables to shed light on the mechanisms of energy dissipation by laser-heated microparticles. The results obtained for aqueous suspensions indicate that the process of laser-induced particle vaporization plays a significant role in the particle energy balance.

\section{Experimental details}

In this paper, all the measurements were carried out with the water suspensions of black submicron particles prepared by diluting and filtering a black gouache paint. Measurements performed with a nephelometer showed an average particle radius of approximately $0.1 \mu \mathrm{m}$. The concentration of particles was controlled by the optical transmittance of a cell with suspension in the visible spectral region (the transmittance was $0.3-0.7$ for a cell thickness of $2 \mathrm{~cm}$ ). During the measurements, the suspension was pumped through the cell; hence, each of the laser pulses interacted with a "fresh" portion of the suspension. All of the experiments were performed at room temperature.

Thermal emission of particles in the suspension was excited by a Q-switched YAG: $\mathrm{Nd}^{3+}$ laser (wavelength $\lambda=1064 \mathrm{~nm}$, pulse duration $\tau=14 \mathrm{~ns}$ FWHM). The laser operation was close to a single-mode regime, with the smooth bell-shaped intensity distribution across the beam axis. The shape of optical pulses was moni- tored with a fast photomultiplier and an oscilloscope with response time of 1.3 and $1 \mathrm{~ns}$, respectively. Other measurements were carried out using the integrating photodetectors. All of the measurements were performed in a single-pulse registration mode.

\section{Results and discussion}

A distinctive feature of LII detected at a fixed wavelength is its nonlinear response to the intensity of laser excitation. Such a behavior is observed both in gases and in condensed matter. A convenient characteristic to describe the degree of nonlinearity of this emission is the following log-log derivative parameter, $\gamma=(\mathrm{d} I / I)(\mathrm{d} F / F)^{-1}$, where $I$ is the power of an LII signal, and $F$ is the laser surface power density. It should be noted that the introduced $\gamma$ parameter does not depend on the measurement units of $I$ and $F$. This is especially suitable for measurements performed in arbitrary units. The experiments show $[10,20]$ that the $\gamma$-parameter depends on both the wavelength and $F$.

The results of measuring $\gamma(F)$ in a water suspension at a wavelength of $500 \mathrm{~nm}$ are presented in Fig. 1. As seen, $\gamma$ abruptly decreases with increase in $F$. However, a plateau typical of LII in gases (i.e. the range of $\gamma=0$ ) is not observed in the suspension.

The fact that $\gamma$ decreases with increase in $F$ is not surprising. Following the above-mentioned assumption that thermal conduction into the surrounding medium is the main mechanism of particle energy loss, a simple approximate relation is derived [10]:

$T \approx \xi F$,

where $T$ is the particle temperature, and $\xi$ is a constant. As $T$ and $F$ are interrelated according to (1), Planck's formula yields the expression

$$
\gamma=\frac{h c / \lambda k_{\mathrm{B}} \xi F}{1-\exp \left(-h c / \lambda k_{\mathrm{B}} \xi F\right)}
$$

where $h$ is the Planck constant, $k_{\mathrm{B}}$ is the Boltzmann constant, and $c$ is the speed of light. The experimental dependence $\gamma(F)$ can be approximated with expression (2) using a single fitting parameter $\xi$. The appropriate graph is given in Fig. 1, curve 1. At first sight, the agreement between the calculated and experimental results seems to be satisfactory. However, the appropriate particle temperatures estimated from (1) at high levels of laser excitation reach the value of $10^{5} \mathrm{~K}$ (see Fig. 2, curve 1), which is apparently far from reality. That is the reason why a new model is required, which could explain experimental results presented in Fig. 1 without presuming unreasonably high temperatures.

Consider the following model of interaction of the laser radiation with spherical microparticles suspended in a transparent liquid (for example, carbon black suspension). The particle energy balance equation can be written as 


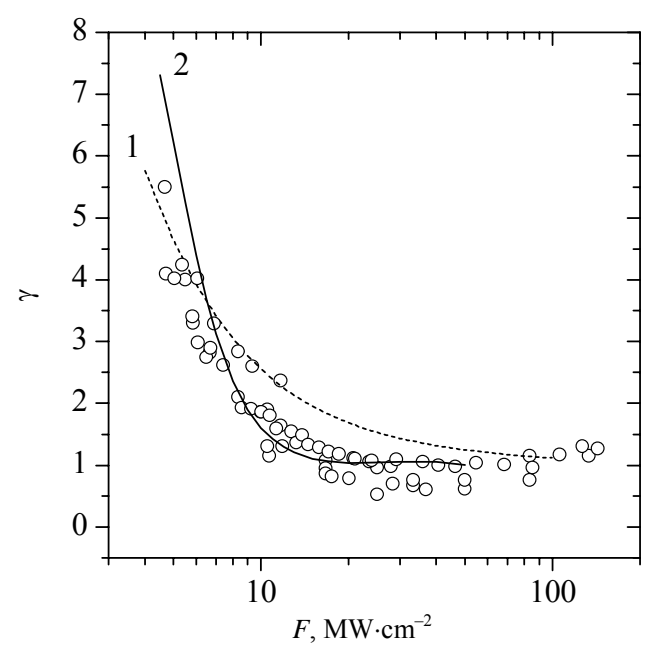

Fig. 1. Parameter of nonlinearity of the thermal emission at a wavelength of $500 \mathrm{~nm}$ as a function of laser surface power density. Circles - experiment, curves - calculations with expression (2) (curve 1) and expressions (3)-(9) (curve 2).

$$
\begin{aligned}
\sigma_{0} F \mathrm{~d} t & =c_{p} m \mathrm{~d} T+\psi_{1}\left(T-T_{0}\right) 4 \pi r_{0}^{2} \mathrm{~d} t+ \\
& +\sigma_{\mathrm{SB}}\left(T^{4}-T_{0}^{4}\right) 4 \pi r_{0}^{2} \mathrm{~d} t+\Lambda_{1}|\mathrm{~d} m|+\Lambda_{2} \mathrm{~d} M,
\end{aligned}
$$

where $\sigma_{0}$ is the particle absorption cross-section, $c_{p}$ is the specific heat of a particle material (carbon), $m$ is the particle mass, $r_{0}$ is the particle radius, $\psi_{1}$ is a variable parameter, $T_{0}$ is the temperature of the surrounding medium, $\sigma_{\mathrm{SB}}$ is the Stefan-Boltzmann constant, $\Lambda_{1}$ and $\Lambda_{2}$ are the latent heat of vaporization of a particle and the surrounding liquid, respectively, and $\mathrm{d} M$ is the mass of the liquid vaporized during the time interval $\mathrm{d} t$.

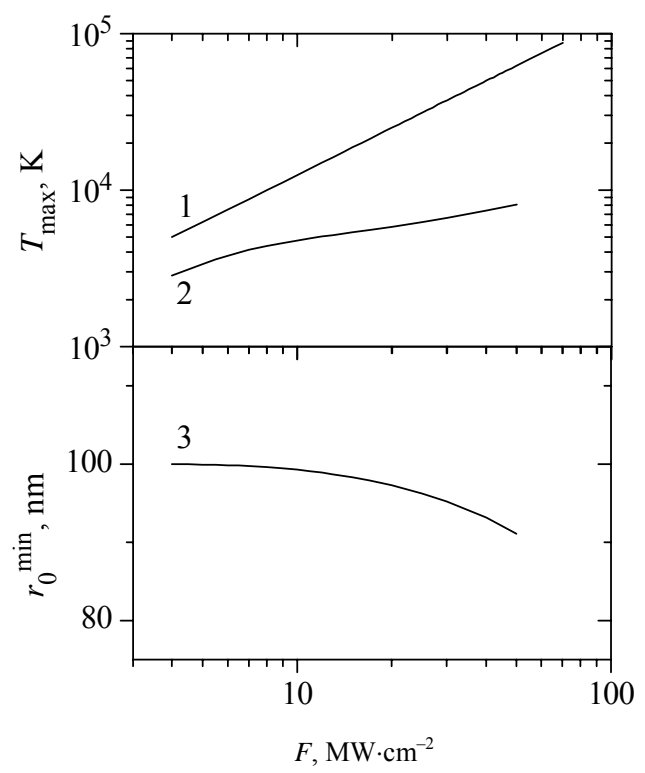

Fig. 2. Calculated maximal particle temperature (curves 1 and 2) and the radius of the particle core at the end of a laser pulse (curve 3 ) as functions of the intensity of laser excitation.
For the process of vaporization of the surrounding water, the following approximate equation can be written [20]:

$G \sigma_{0} F \mathrm{~d} t=\Lambda_{2} \mathrm{~d} M$,

where $G$ is the fraction of the absorbed laser energy which is spent on the vaporization of the surrounding water. Presume that a spherical gaseous shell grows around the particle during a laser pulse. Then the volume of the shell can be written as $V=\frac{4}{3} \pi\left(r^{3}-r_{0}^{3}\right)$, where $r$ is the radius of the core-shell particle that consists of a solid core with the radius $r_{0}$ and a gaseous shell with the thickness $\left(r-r_{0}\right)$. Using the equation of state for an ideal gas, the following approximate equation can be derived:

$\frac{\mathrm{d} r}{\mathrm{~d} t}=\frac{G \sigma_{0} R}{4 \pi p \mu \Lambda_{2}} r^{-2} F(t) T(t)$,

where $R$ is the gas constant, $\mu$ is the molar mass of water, $p$ is the average gas pressure in the shell. It is assumed that the average gas temperature in the shell does not differ significantly from the particle temperature.

The energy, $d W$, lost by a particle due to the thermal radiation in a narrow spectral interval $(\lambda, \lambda+\mathrm{d} \lambda)$ can be represented according to Planck's formula as

$\mathrm{d} W=$ const $\left[\exp \left(\frac{h c}{\lambda k_{\mathrm{B}} T(t)}\right)-1\right]^{-1} r_{0}^{2} \mathrm{~d} t$.

Eq. (6) will be used below for the calculation of optical signals of thermal radiation, in particular for the calculation of the parameter of nonlinearity $\gamma$.

The lack of information about the processes of thermal conduction in the surrounding medium [the second term on the right-hand side of Eq. (3)] and about the vaporization of the particle core [the penultimate term in (3)] brings a significant uncertainty in the calculations. Consequently, the model involves two variable parameters that are fitted for the best agreement between the calculated and experimental data. The first variable parameter mentioned is $\psi_{1}$ in Eq. (3).

The particle mass change caused by vaporization can be approximated as

$\mathrm{d} m=-$ const $\cdot \sigma_{0} F \mathrm{~d} t$,

where the absorption cross section depends on the core radius according to the Mie theory

$\sigma_{0}=$ const $\cdot r_{0}^{2}$.

Therefore, the following equation can be written for a laser-induced decrease of the core radius:

$\mathrm{d} r_{0}=-\psi_{2} F \mathrm{~d} t$

where $\psi_{2}$ is the second variable parameter of the calculations.

Thus, the coupled equations (3), (5), (6), and (9) can be solved by numerical integration with respect to 
$T(t), \quad r(t), \quad W(t)$, and $r_{0}(t)$, and the parameter of nonlinearity can be calculated as a function of laser intensity, $\gamma(F)$. The calculations were carried out for the thermal radiation at a wavelength of $500 \mathrm{~nm}$. The laser pulse with a duration of 14 ns was approximated by the Gauss function. The cross-beam laser intensity distribution was considered as uniform. The computer code allowed for the particle vaporization [Eq. (9) and the penultimate term in (3)] only at particle temperatures above $4000 \mathrm{~K}$.

The calculated data are plotted in Fig. 1, curve 2. As is seen from the figure, a good agreement with the experimental data is obtained. In addition, the estimated maximal temperature of the particle, $T_{\max }$, exceeds the temperature of carbon vaporization by a factor of at most two. The typical calculated functions $T_{\max }(F)$ and $r_{0}{ }^{\min }(F)$ are presented in Fig. 2, where $r_{0}{ }^{\text {min }}$ is the particle core radius at the end of a laser pulse. We note that the calculated radius of a core-shell particle, $r(t)$, is in agreement with the optical limiting experimental data given in [20].

As seen from Fig. 2, curve 3, the decrease of the carbon particle radius due to the particle vaporization is expected to be up to $5-10 \%$ at the end of a laser pulse. This circumstance can affect the LII emission decay at high levels of laser excitation. Assuming that the thermal conduction to the environment is the main mechanism of particle energy dissipation after a laser pulse, the time of particle temperature relaxation is proportional to the particle radius [10]. Thus, a reduction of the LII lifetime at high levels of laser excitation can be expected. To verify this assumption, we investigated the shape of LII pulses as a function of the intensity of laser excitation. Typical oscillograms are given in Fig. 3. One can see that the leading edge of an LII pulse is distinctly shorter than that of the laser pulse, as it naturally should be for the nonlinear emission with the index of nonlinearity more than unity. The trailing edge of an LII pulse is essentially longer than that of the laser pulse due to the particle slow cooling.

The results of measuring the FWHM of LII pulses, $\tau_{\mathrm{LII}}$, are presented in Fig. 4 . The figure shows that, in the range of $F<20 \mathrm{MW} \cdot \mathrm{cm}^{-2}, \tau_{\mathrm{LII}}$ significantly decreases with increase in $F$, which is consistent with the abovepresented considerations concerning the decrease of a particle size. In addition, it was observed that, as the suspension is excited by tandem laser pulses with a time interval of about $0.1 \mathrm{~s}$, the second LII pulse appears to be a few percent shorter than the first one. This fact can be also explained by a reduction of the particle radius due to vaporization.

Numerical calculations of $\tau_{\text {LII }}$ were carried out using the system of equations (3), (5), (6), and (9). The calculation results are presented in Fig. 4 as a solid curve. As seen, the satisfactory agreement is obtained with the experimental data in the range of $F<$ $20 \mathrm{MW} \cdot \mathrm{cm}^{-2}$. It should be noted that, due to the presence of the variable parameter $\psi_{1}$, only relative changes of the LII lifetime can be calculated.

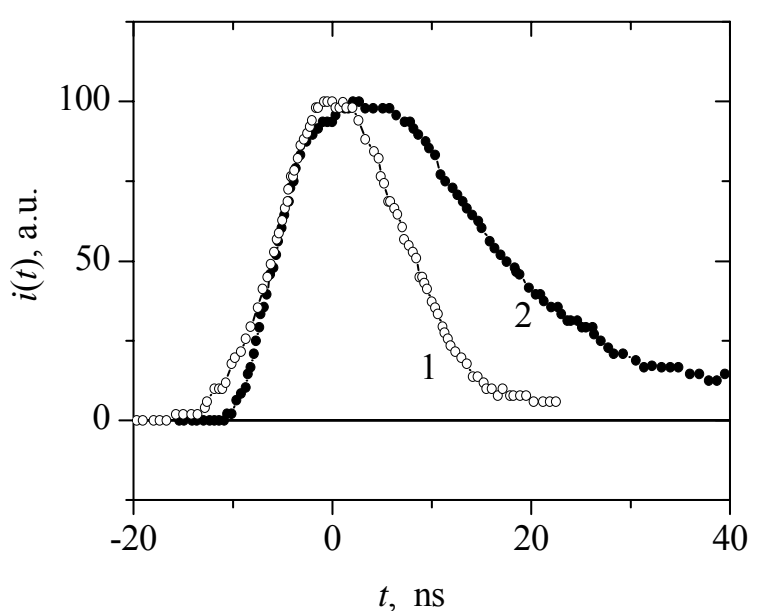

Fig. 3. Typical oscillograms of a laser pulse (1) and the thermal emission (2).

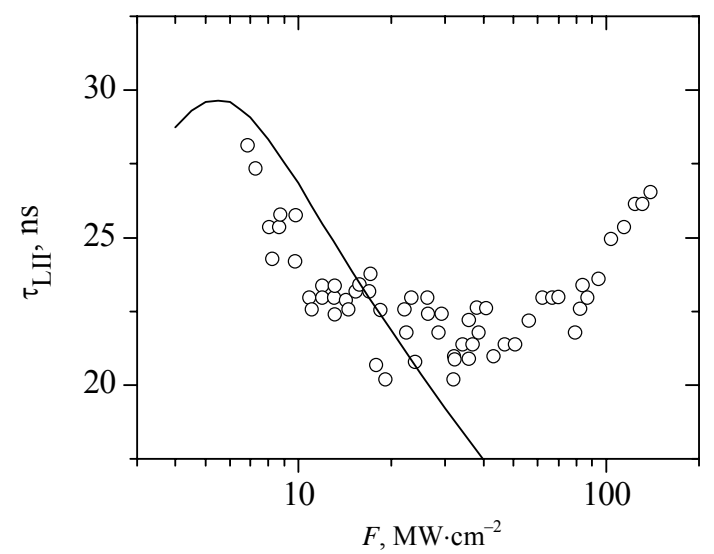

Fig. 4. Thermal emission lifetime in a water suspension as a function of the intensity of laser excitation. Circlesexperiment, curve - the result of calculations.

Thus, Figs. 1 and 4 show the agreement between the results of calculations and the experimental data obtained at moderate levels of laser excitation, which argues in favor of the proposed model of the interaction of light-absorbing microparticles with laser radiation. As regards the characteristics of LII in the range of high intensities of excitation, $F>20 \mathrm{MW} \cdot \mathrm{cm}^{-2}$, the model fails to describe the experimentally observed increase of $\gamma$ and $\tau_{\text {LII }}$. A plausible supposition is that the mechanism of the interaction of particles with laser light changes significantly at high levels of excitation.

\section{Concluding remarks}

In conclusion, some remarks should be made concerning the proposed model of the interaction of light-absorbing particles with laser radiation. It is obvious that the proposed model is simplified, and it does not take into account a number of characteristics, both known and 
unknown, of the processes considered. First, the model does not take into consideration the processes of laser plasma formation in the vicinity of a particle. Second, the quick growth of a gaseous shell is accompanied by the generation of acoustic waves [21,22], which can be an additional mechanism of particle energy loss. The efficiency of this mechanism is difficult to estimate numerically. Third, the model does not take into account the behavior of optical characteristics of a core-shell particle during its growth. This can cause the time dependence of the absorption cross-section to be more complicated than within the geometric approach [8]. In addition, physical characteristics of materials at high temperatures are known approximately. Nevertheless, despite the mentioned limitations of the proposed model, it provides a satisfactory explanation for the characterristics of LII in aqueous suspensions of submicron blackbody particles considered in this paper.

\section{References}

1. A.C. Eckbreth, Effects of laser-modulated particulate incandescence on Raman scattering diagnostics // J. Appl. Phys. 48(11), p. 4473-4479 (1977).

2. R.L. Vander Wal and K.J. Weiland, Laser-induced incandescence: development and characterization towards a measurement of soot-volume fraction // Appl. Phys. B 59, p. 445-452 (1994).

3. F. Cignoli, S. Benecchi, G. Zizak, Time-delayed detection of laser-induced incandescence for the two-dimensional visualization of soot in flames // Appl. Opt. 33(24), p. 5778-5782 (1994).

4. R.L. Vander Wal, M.Y. Choi, K.-O. Lee, The effects of rapid heating of soot: implications when using laser-induced incandescence for soot diagnostics // Combustion and Flame 102, p. 200-204 (1995).

5. C.R. Shaddix, K.C. Smyth, Laser-induced incandescence measurements of soot production in steady and flickering methane, propane, and ethylene diffusion flames // Combustion and Flame 107, p. 418-452 (1996).

6. R.L. Vander Wal, Laser-induced incandescence: detection issues // Appl. Opt. 35(33), p. 6548-6559 (1996).

7. G.S. Eom, C.W. Park, Y.H. Shin et al., Size determination of nanoparticles in low-pressure plasma with laser-induced incandescence technique // Appl. Phys. Lett. 83(6), p. 1261-1263 (2003).

8. S. Zelensky, Laser-induced heat radiation in borate glass // J. Phys.: Condens. Matter. 10, p. $7267-$ 7272 (1998).
9. K. Mansour, M.J. Soileau, E.W. Van Stryland, Nonlinear optical properties of carbon-black suspensions (ink) // J. Opt. Soc. Amer. B 9(7), p. 1100-1109 (1992).

10. S. Zelensky, Laser-induced heat radiation of suspended particles: a method for temperature estimation // J. Opt. A: Pure Appl. Opt. 1, p. 454458 (1999).

11. S. Zelensky, Laser-induced incandescence of suspended particles as a source of excitation of dye luminescence // J. Luminescence 104, p. 27-33 (2003).

12. P. Roura, J. Costa, Radiative thermal emission from silicon nanoparticles: a reversed story from quantum to classical theory // European J. Phys. 23, p. 191-203 (2002).

13. K.M. Nashold, D.P. Walter, Investigations of optical limiting mechanisms in carbon particle suspensions and fullerene solutions // J. Opt. Soc. Amer. B 12(7), p. 1228-1237 (1995).

14. X. Sun, R.Q. Yu, G.Q. Xu et al. Broadband optical limiting with multiwalled carbon nanotubes // Appl. Phys. Lett. 73(25), p. 3632-3634 (1998).

15. P. Chen, X. Wu, X. Sun et al. Electronic structure and optical limiting behavior of carbon nanotubes // Phys. Rev. Lett. 82(12), p. 2548-2551 (1999).

16. X. Sun, Y. Xiong, P. Chen et al., Investigation of an optical limiting mechanism in multiwalled carbon nanotubes // Appl. Opt. 39(12), p. 19982001 (2000).

17. Z. Jin, L. Huang, S.H. Goh et al., Size-dependent optical limiting behavior of multi-walled carbon nanotubes// Chem. Phys. Lett. 352, p. 328-333 (2002).

18. S.K. Tiwari, M.P. Joshi, M. Laghate, et al., Role of host liquid in optical limiting in ink suspensions // Optics \& Laser Technology. 34, p. 487-491 (2002).

19. S.K. Tiwari, M.P. Joshi, S. Nath et al., Salt-induced aggregation and enhanced optical limiting in carbon-black suspensions // J. Nonlinear Opt. Phys. Mater. 12(3), p. 1-5 (2003).

20. S.E. Zelensky, Self-induced attenuation of pulsed laser radiation in an aqueous suspension of submicron light-absorbing particles // J. Phys.: Condens. Matter. 15, p. 6647-6657 (2003).

21. H. Lowen, P.A. Madden, A microscopic mechanism for shock-wave generation in pulsed-laserheated colloidal suspensions // J. Chem. Phys. 97(11), p. 8760-8766 (1992).

22. A.C. Beveridge, T.E. McGrath, G.J. Diebold et al., Photoacoustic shock generation in carbon suspensions // Appl. Phys. Lett. 75(26), p. 4204 4206 (1999). 